\title{
Assessment of static bed height and particle diameter on the hydrodynamic behavior of a spouted bed
}

\author{
S. A. Tabatabaei ${ }^{1}$, N. Mahinpey ${ }^{1}$, C. J. Lim $^{2}$ \& E. Esmaili ${ }^{1}$ \\ ${ }^{1}$ Department of Chemical and Petroleum Engineering, \\ University of Calgary, Canada \\ ${ }^{2}$ Department of Chemical and Bio-Resources Engineering, \\ University of British Columbia, Canada
}

\begin{abstract}
Spouted beds are widely used as a gas-solid contactor in various physical and chemical processes. In order to improve the design of spouted beds for efficient gas-solid contact, a better understanding of the complex flow field of granular solids is required. In this paper, the computational fluid dynamics technique was used to simulate the gas-solid two-phase flow in a slot-rectangular spouted bed. The two-phase Eulerian-Eulerian granular model was studied. A twodimensional computer simulation using FLUENT commercial software was performed to investigate the effects of physical parameters on the hydrodynamic behavior of slot-rectangular spouted beds. The fountain height was evaluated for various superficial gas velocities, bed heights, and solid particle sizes. The numerical results of the fountain height were in good agreement with the experimental data reported elsewhere.

Keywords: spouted bed, slot-rectangular, hydrodynamics, two-phase flow, CFD.
\end{abstract}

\section{Introduction}

Spouted beds are used as gas-solid contactors to provide efficient mixing and large gas-solid contact areas for coarse granular materials. They have been widely used in various physical and chemical processes, such as drying, granulation, agglomeration, coating, coal gasification, and combustion. 


\section{Nomenclature}

\begin{tabular}{|cl|}
\hline$C_{d}$ & Single particle drag function, dimensionless \\
$d_{s}$ & Solid diameter, m \\
$e$ & Restitution coefficient, dimensionless \\
$\mathrm{d}_{\mathrm{p}}$ & Particle diameter \\
$\mathrm{H}_{\mathrm{b}}$ & Static bed height \\
$\mathrm{u}$ & Air inlet Velocity \\
$g$ & Acceleration due to gravity, $\mathrm{m} / \mathrm{s}^{2}$ \\
$g_{0}$ & Radial distribution coefficient, dimensionless \\
$K_{s g}$ & Gas/solid momentum exchange coefficient, dimensionless \\
$P$ & Pressure, Pa \\
$P_{s}$ & Solid pressure, Pa \\
$\vec{V}$ & Velocity, m/s \\
$R e$ & Reynolds number, dimensionless \\
$k_{\Theta_{s}}$ & Conductivity of granular temperature, $\mathrm{kg} /(\mathrm{m} . \mathrm{s})$ \\
$\gamma_{\Theta_{s}}$ & Dissipation of granular temperature, $\mathrm{kg} /\left(\mathrm{m} . \mathrm{s}^{3}\right)$ \\
& \\
$\mathrm{Greek}$ & \\
$\rho$ & Dymbols \\
$\Theta_{s}$ & Granular temperature, $\mathrm{m}^{2} / \mathrm{s}^{2}$ \\
$\overline{\bar{\tau}}$ & Stress tensor, Pa \\
$\mu$ & Shear viscosity, kg/sm \\
$\lambda$ & Bulk viscosity, kg/sm \\
$\varepsilon$ & Volume fraction, dimensionless \\
Subscripts \\
$g$ & Gas \\
$s$ & Solid \\
\hline & \\
\hline
\end{tabular}

A conventional spouted bed consists of a cylindrical column and a conical base with a circular orifice at the center of the conical base. The bed of solid particles is spouted by the injection of a high velocity fluid through the orifice. A spouted bed is typically formed from three distinct regions: (1) a central spout with upward gas-solid flow, (2) an annulus region of downward granular flow, and (3) a fountain above the bed surface [1]. The volume fraction of solid particles varies from low values in the spout region to large values in the annulus, where the particles fall vertically downward and radially inward, leading to a complex recirculation pattern.

Conventional cylindrical-conical spouted beds suffer from the difficulties of scale-up [2]. A rectangular cross-sectional spouted bed was suggested to overcome these limitations [3-5]; however, significant three-dimensional effects were then found as the column thickness increased [6, 7]. Therefore, the term "slot-rectangular" is preferred for these types of spouted beds. 
The idea of a two-dimensional spouted bed focuses on using a rectangular cross-sectional column with a large column width to thickness ratio and featuring two vertical parallel plates. The scale-up of such columns has been suggested to be easily achieved by increasing the thickness [4]. However, three-dimensional instabilities have been shown as an issue for scale-up [8, 9]. Slot-rectangular spouted beds can be utilized in similar applications as those proposed for conventional spouted beds. However, investigations on the application of slotrectangular spouted beds have been mainly limited to the coating of particles. Numerous studies on the flow hydrodynamics, stability and scale-up of slotrectangular spouted beds have been reported [3-13].

A better understanding of the complex flow field of granular solids in spouted beds is required to obtain optimal mixing for their design improvement. Considering the advances in computer simulations, numerical simulation techniques have become a powerful and popular tool for acquiring detailed information about the gas-solid two-phase flow without influencing it [14]. Computational fluid dynamics (CFD) is the most common numerical technique to simulate multiphase flows. The use of CFD simulations for spouted beds significantly reduces the need for experimentation by providing information on the flow pattern, scale-up and optimization for process design [15-19].

Zhonghua and Mujumdar [15] developed a heat and mass transfer CFD model to study the drying characteristics of particulate solids in axisymmetric spouted beds. Their calculated particle velocities and concentrations were in agreement with previous experimentations reported by He et al. [1]. They also studied the gas-particle flow behavior in a cylindrical spouted bed and a three-dimensional spout-fluid bed using the Eulerian-Eulerian two-fluid modeling approach [16]. Du et al. [17] assessed the influences of incorporating several drag models into the two-fluid model on the CFD simulation of spouted beds. Their results of hydrodynamic properties were compared with the experimental results reported by He et al. [1]. Their investigation showed that drag models have critical effects on the CFD predictions in spouted beds.

Scientific literature related to numerical simulations of slot-rectangular spouted beds is scarce. Zhao et al. [18] investigated the dynamics of granular particles in a two-dimensional spouted bed with draft plates by employing both particle image velocimetry (PIV) measurement and discrete element method (DEM) simulation. Their DEM-CFD simulations provided a good prediction of the longitudinal profile of the particle vertical velocity along the bed centerline, especially during the rapid acceleration stage at the lower part of the spout. Zhao et al. [19] performed DEM simulation together with a low Reynolds number $k-\varepsilon$ turbulence model for the fluid phase, in order to study the periodic spouting of granular solids in a two-dimensional spouted bed. The simulation results corresponded precisely with the data of PIV experiments, including fluid flow fields, time-averaged particle velocity distributions, and spout shape. The simulations yielded the predictions of an unstable spout regime, characterized as a periodic upward-moving particle jet.

In the present study, the simulation of the gas-solid flows in a slot-rectangular spouted bed based on the experimental setup of Dogan et al. [6] was performed 
using a two-phase Eulerian-Eulerian granular model. The interfacial drag force was described using the adjusted Di Felice model [20]. A CFD commercial software package, FLUENT, was used for the two-dimensional computer simulation. The effects of system parameters on the hydrodynamic behavior of the slot-rectangular spouted beds were studied. The results are presented in terms of contours of the solid volume fraction. The fountain height was evaluated for various superficial gas velocities, bed heights, and solid particle sizes. The numerical results on the fountain height were compared with experimental data from Dogan et al. [6].

\section{Description of the model}

The structure of the bed used in the current study is shown in Figure 1. The model had a two-dimensional rectangular configuration that attempted to replicate that of the experimental work of Dogan et al. [6]. The height of the column and the width of the column at the upper and lower sections were $\delta=70$ $\mathrm{cm}, \alpha=15 \mathrm{~cm}$ and $\gamma=4 \mathrm{~cm}$, respectively. The internal angle of the lower section, $\theta$, was $30^{\circ}$. Slots with widths $(\lambda)$ of 6,10 and $20 \mathrm{~mm}$ for air entry and static bed heights $\left(H_{b}\right)$ of 11,16 and $21 \mathrm{~cm}$ were considered in the simulations. The particles used in the simulation had the same density $\left(\rho_{S}\right)$ of $2520 \mathrm{~kg} / \mathrm{m}^{3}$ but four different diameters $\left(d_{p}\right): 0.86,1.44,2.28$ and $3.77 \mathrm{~mm}$. The restitution coefficients between the particles and the maximum packing limit had values of 0.95 and 0.63 , respectively. At the beginning, the solid in the bed was at its maximum packing limit. The density and viscosity of the inlet air were $1.225 \mathrm{~kg} / \mathrm{m}^{3}$ and $1.7894 \times 10^{-5} \mathrm{~kg} / \mathrm{m} . \mathrm{s}$, respectively.

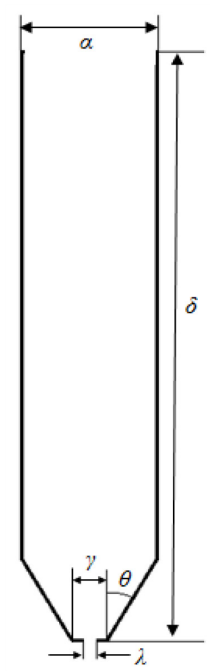

Figure 1: Schematic of the problem configuration. 


\section{Governing equations}

In this study, the Eulerian-Eulerian two-fluid model, which treats the gas and solid phases as fully interpenetrating continua, was used to simulate the hydrodynamics of a two-dimensional spouted bed. The balance in volume fractions of all contributing phases gives:

$$
\sum_{q=s, g} \varepsilon_{q}=1
$$

where $s$ and $g$ denote the solid and gas phases, respectively. The continuity equation for phase $q$ is as follows:

$$
\frac{\partial}{\partial t}\left(\varepsilon_{q} \rho_{q}\right)+\nabla \cdot\left(\varepsilon_{q} \rho_{q} \vec{V}_{q}\right)=0,
$$

in which $q$ takes both $s$ and $g$ indices, in order to conserve the mass in the solid and gas phases, respectively. The conservation of momentum in the solid and gas phases are given in Eqs. (3a) and (3b), respectively:

$$
\begin{gathered}
\frac{\partial}{\partial t}\left(\varepsilon_{g} \rho_{g} \vec{V}_{g}\right)+\nabla \cdot\left(\varepsilon_{g} \rho_{g} \vec{V}_{g} \vec{V}_{g}\right) \\
\quad=\nabla \cdot \overline{\bar{\tau}}_{g}-\varepsilon_{g} \nabla P+\varepsilon_{g} \rho_{g} g+K_{s g}\left(\vec{V}_{s}-\vec{V}_{g}\right), \\
\frac{\partial}{\partial t}\left(\varepsilon_{s} \rho_{s} \vec{V}_{s}\right)+\nabla \cdot\left(\varepsilon_{s} \rho_{s} \vec{V}_{s} \vec{V}_{s}\right) \\
=\nabla \cdot \overline{\bar{\tau}}_{s}-\nabla P_{s}-\varepsilon_{s} \nabla P+\varepsilon_{s} \rho_{s} g+K_{s g}\left(\vec{V}_{s}-\vec{V}_{g}\right) .
\end{gathered}
$$

The equations for the stress tensor are governed by Eqs. (4)-(9):

$$
\begin{gathered}
\overline{\bar{\tau}}_{\mathrm{g}, \mathrm{eff}}=\varepsilon_{\mathrm{g}}\left(\overline{\bar{\tau}}_{\mathrm{g}}+\overline{\bar{\tau}}_{\mathrm{t}}\right), \\
\overline{\bar{\tau}}_{g}=\mu_{g}\left(\nabla \vec{V}_{g}+\left(\nabla \vec{V}_{g}\right)^{T}\right)-\frac{2}{3} \mu_{g} \overline{\bar{I}} \cdot \nabla \cdot \vec{V}_{g}, \\
\overline{\bar{\tau}}_{t}=\mu_{t}\left(\nabla \vec{V}_{g}+\left(\nabla \vec{V}_{g}\right)^{T}\right)-\frac{2}{3} \mu_{t} \overline{\bar{I}} \cdot \nabla \cdot \vec{V}_{g}-\frac{2}{3} \varepsilon_{g} \rho_{g} k \overline{\bar{I}}, \\
\overline{\bar{\tau}}_{s}=\varepsilon_{s} \mu_{s}\left(\nabla \vec{V}_{s}+\left(\nabla \vec{V}_{s}\right)^{T}\right)+\varepsilon_{s}\left(\lambda_{s}+\frac{2}{3} \mu_{s}\right) \nabla \cdot \vec{V}_{s} \overline{\bar{I}}, \\
\mu_{s}=\frac{4}{5} \varepsilon_{s}^{2} \rho_{s} d_{s} g_{0}(1+e) \sqrt{\frac{\theta}{\pi}}+\frac{\frac{5 \sqrt{\pi}}{48} \rho_{s} d_{s} \sqrt{\Theta}}{(1+e) g_{0}}\left[1+\frac{4}{5} g_{0} \varepsilon_{s}(1+e)\right]^{2}, \\
\lambda_{s}=\frac{4}{3} \varepsilon_{s}^{2} \rho_{s} d_{s} g_{0}(1+e) \sqrt{\frac{\Theta}{\pi}} .
\end{gathered}
$$

The granular temperature is described by Eqs. (10)-(14):

$$
\begin{gathered}
g_{0}=\frac{3}{5}\left[1-\left(\frac{\varepsilon_{s}}{\varepsilon_{s, \max }}\right)^{\frac{1}{3}}\right]^{-1}, \\
\frac{3}{2}\left[\frac{\partial}{\partial t}\left(\varepsilon_{s} \rho_{s} \Theta_{s}\right)+\nabla \cdot\left(\varepsilon_{s} \rho_{s} \vec{V}_{s} \Theta_{s}\right)\right]=\overline{\bar{\tau}}_{s}: \nabla \vec{V}_{s}-\nabla \cdot q_{s}-\gamma_{\Theta_{s}}-3 K_{s g} \Theta_{s}, \\
q_{s}=k_{\Theta_{s}} \nabla \Theta_{s},
\end{gathered}
$$




$$
\begin{gathered}
k_{\Theta_{s}}=\frac{150 d_{s} \rho_{s} \sqrt{\Theta_{s} \pi}}{384(1+e) g_{0}}\left[1+\frac{6}{5}(1+e) \varepsilon_{g} g_{0}\right]^{2} \\
+2 d_{s} \rho_{s} \varepsilon_{s}{ }^{2}(1+e) g_{0} \sqrt{\frac{\Theta_{s}}{\pi}}, \\
\gamma_{\Theta_{s}}=\frac{12\left(1-e^{2}\right) g_{0}}{d_{s} \sqrt{\pi}} \rho_{s} \varepsilon_{s}^{2} \sqrt{\Theta_{s}^{3}} .
\end{gathered}
$$

The interphase momentum exchange is computed using Eqs. (15)-(20):

$$
\begin{gathered}
K_{s g}=\frac{3}{4} C_{D} \frac{\varepsilon_{s} \rho_{g}}{d_{s}}\left|\vec{V}_{s}-\vec{V}_{g}\right| f\left(\varepsilon_{s}\right), \\
f\left(\varepsilon_{s}\right)=\left(1-\varepsilon_{s}\right)^{-x}, \\
x=P-Q \cdot \exp \left[-(1.5-\beta)^{2} / 2\right], \\
\beta=\log \left(R e_{s}\right) .
\end{gathered}
$$

where $P$ and $Q$ are adjusted to satisfy the following relations:

$$
\begin{gathered}
\varepsilon_{s}\left(\rho_{s}-\rho_{g}\right) g=\frac{K_{s g}}{\varepsilon_{g}}\left|\vec{V}_{s}-\vec{V}_{g}\right|, \\
\varepsilon_{s, m f}\left(\rho_{s}-\rho_{g}\right) g=\frac{K_{s g}}{\varepsilon_{g, m f}} U_{m f}^{\text {experiment }} .
\end{gathered}
$$

\section{Numerical simulation}

Commercial CFD software FLUENT was selected to run the numerical simulations. The set of governing equations were discretized using the finite volume method. The Phase-Coupled Semi-Implicit Method for Pressure-Linked Equations (PC-SIMPLE) provided the pressure-velocity coupling. The QUICK and second-order upwind discretization schemes discretized the partial differential equations of volume fraction and momentum.

The two-fluid Eulerian-Eulerian approach was applied. This technique considers separate sets of conservation of mass and momentum for each continuous and incompressible phase. In order to include the momentum transfer between the phases, the interfacial drag force was implemented using the adjusted Di Felice model [20].

The 2D computational meshed domain was composed of unstructured triangular cells in the cone base and structured square cells in the rest of the domain. Meshing software Gambit 2.13 generated the computational grids. The grid used in the domain discretization is shown in Figure 2. The generated grids had node spacing larger than the particle diameter, namely $0.0025 \mathrm{~m}$. The mesh independency of the results was checked with variation of the number of mesh elements.

A fixed time step size of $1 \times 10^{-3} \mathrm{~s}$ was applied. A maximum of 50 iterations were used for each time step, and the convergence criteria of the solutions were assumed at scaled residuals of smaller than $10^{-3}$. A value of 0.2 for all under- 


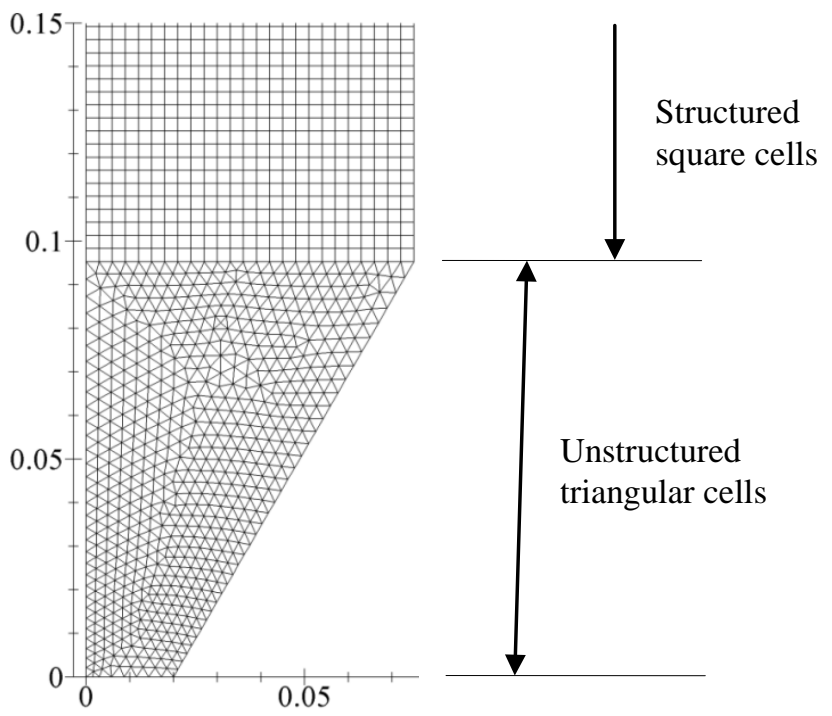

Figure 2: $\quad$ Computational mesh.

relaxation factors was chosen. Two seconds of flow time simulation was necessary to ensure a statistically steady-state fountain height.

In order to reduce computational times, the symmetry boundary condition was assumed along the axis of symmetry; therefore, the required simulations were carried out in half of the two-dimensional domain. The boundary condition at the entrance was considered as a velocity inlet with a uniform velocity profile. A pressure outlet boundary condition was applied at the outlet of the system. On the walls of the spouted bed, a no-slip boundary condition was assumed.

\section{Results and discussion}

The simulated results of gas-solid flows in a slot-rectangular spouted bed are presented for various values of the effective parameters. In order to validate the current model, the numerical results of the fountain height were compared with the experimental data reported by Dogan et al. [6]. Figure 3 shows the results of the fountain height for different superficial air velocities in the case of $H_{b}=11$ $\mathrm{cm}, d_{p}=0.86 \mathrm{~mm}$ and $\lambda=6 \mathrm{~mm}$. This figure confirms the agreement between the results of the numerical simulation and the reported experimental results.

Figures 4, 5 and 6 present the values of the fountain height versus the superficial air velocity for different values of slot width, static bed height and particle diameter, respectively. In addition to the variable parameter, the fixed values of the system parameters for these figures were: $H_{b}=11 \mathrm{~cm}$ and $d_{p}=1.44$ $\mathrm{mm}$ for Figure $4, \lambda=6 \mathrm{~mm}$ and $d_{p}=0.86 \mathrm{~mm}$ for Figure 5 , and $\lambda=6 \mathrm{~mm}$ and $H_{b}=16 \mathrm{~cm}$ for Figure 6 . These figures show that the fountain height increases with increasing in superficial air velocity. 


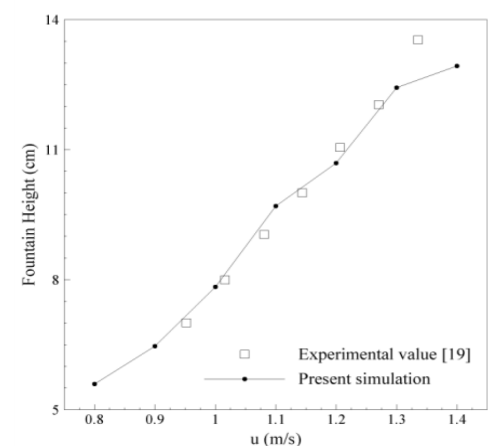

Figure 3: Comparison of this study's numerical results of fountain height and the experimental data of Dogan et al. [6].

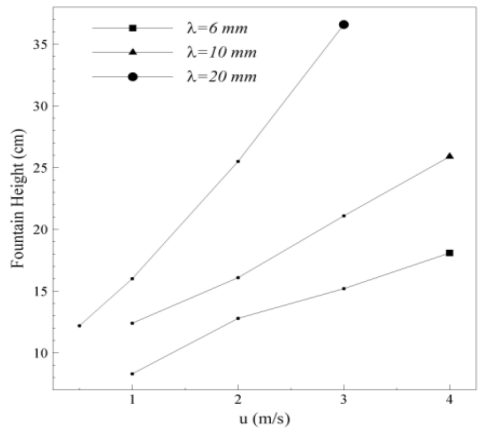

Figure 4: Fountain height versus superficial air velocity for different slot widths $\left(H_{b}=11 \mathrm{~cm}\right.$ and $d_{p}=1.44$ $\mathrm{mm})$.

Figure 4 shows that fountain height increased with increases in slot width at constant values of superficial air velocity. As a result, with a smaller slot width, a higher superficial gas velocity is required to reach the same height of the fountain. Figure 4 also reveals that the rate of increase in the fountain height as a function of superficial air velocity increased with increases in slot width.

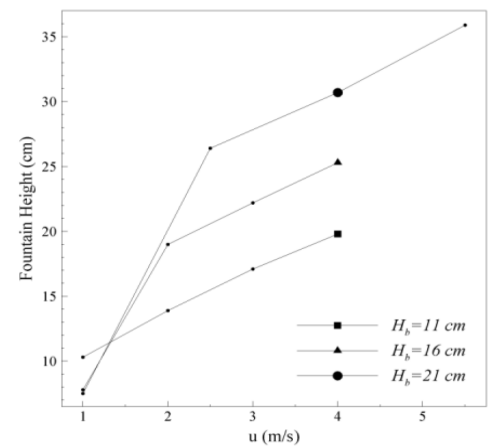

Figure 5: Fountain height versus superficial air velocity for different static bed heights $(\lambda=6 \mathrm{~mm}$ and $\left.d_{p}=0.86 \mathrm{~mm}\right)$.

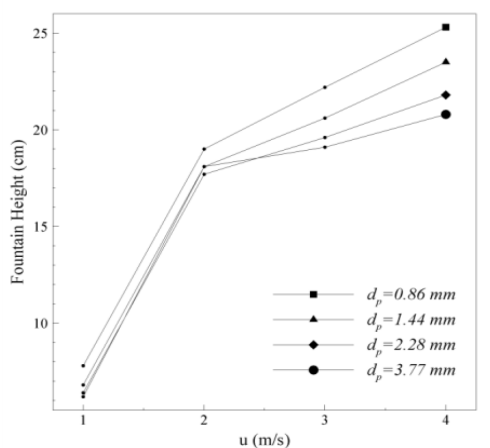

Figure 6: Fountain height versus superficial air velocity for different particle diameters $(\lambda=6 \mathrm{~mm}$ and $\left.H_{b}=16 \mathrm{~cm}\right)$.

In Figure 5, two distinct regions can be distinguished for the variations in fountain height with respect to the static bed height at constant values of superficial air velocity. When the superficial air velocity was sufficiently low, the air-induced force vanished for high values of static bed height. This finding suggests that the fountain height had a decreasing trend with increases in the 
static bed height for low values of superficial air velocity, and vice versa. Figure 5 also reveals that the rate of increase in the fountain height as a function of the superficial air velocity at a constant static bed height decreased with increases in the superficial air velocity.

Ignoring the inconsistency that occurred at $d_{p}=3.77 \mathrm{~mm}$ and superficial air velocity $(u)=2 \mathrm{~m} / \mathrm{s}$, Figure 6 shows that the fountain height decreased with increases in the particle diameter at constant values of superficial air velocity. This implies that, with a larger particle diameter, a higher superficial gas velocity is required to obtain the same fountain height. Figure 6 also suggests that the rate of increase in the fountain height as a function of the superficial air velocity at a constant size of particle diameter decreased with increases in the superficial air velocity. Although the fountain height decreased with increases in the particle diameter for the condition cited in Figure 6, it may increase with particle diameter at different conditions of $\lambda$ and $H_{b}$ [6].

The solid volume fraction contours after 2 seconds of real-time simulation at different superficial air velocities are presented in Figures 7 and 8 for static bed heights of 11 and $21 \mathrm{~cm}$, respectively. In these figures, the three different parts of the spout bed - the annulus, spout and fountain - can be distinguished. The highest concentration of solid particles were seen in the annulus, which is practically constant. Lower values of solid volume fractions could be found in the fountain and the spout regions. The formation of the fountain only occurred after exceeding a certain value of gas velocity for the spouted beds in a specific working condition.

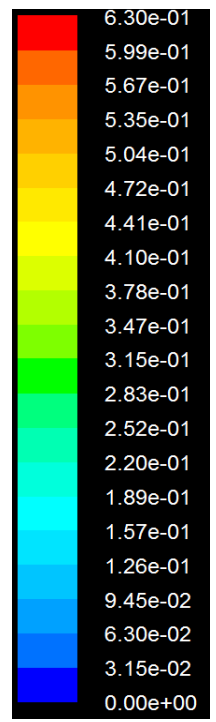

$u=$

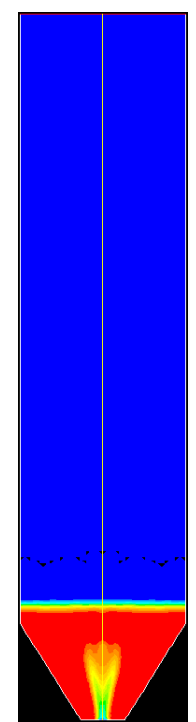

$1 \mathrm{~m} / \mathrm{s}$

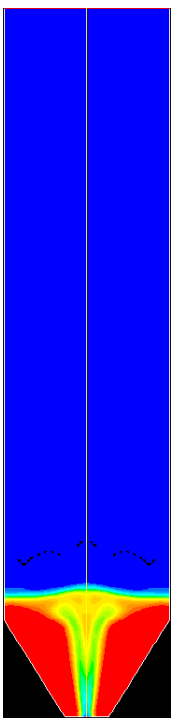

$2 \mathrm{~m} / \mathrm{s}$

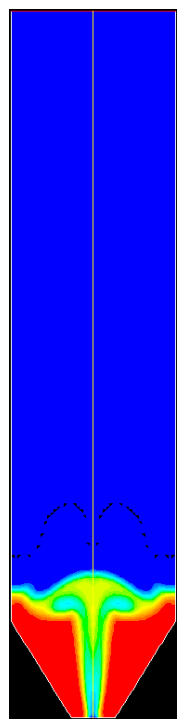

$3 \mathrm{~m} / \mathrm{s}$

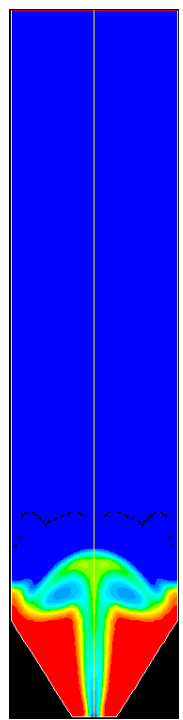

$4 \mathrm{~m} / \mathrm{s}$

Figure 7: Contours of the solid volume fraction for different superficial gas velocities ( $\lambda=6 \mathrm{~mm}, H_{b}=11 \mathrm{~cm}$ and $d_{p}=2.28 \mathrm{~mm}$ ). 
The flow regimes in Figure 7 were identified as an internal jet for the case of $u=1$ and as spouting for $u=2,3$ and 4 . The flow regimes in Figure 8 were identified as an internal jet for the case of $u=1$ and as incoherent spouting for $u=3$ and 4 , and as a transition between a jet-in-fluidized-bed and incoherent spouting for $u=2$.

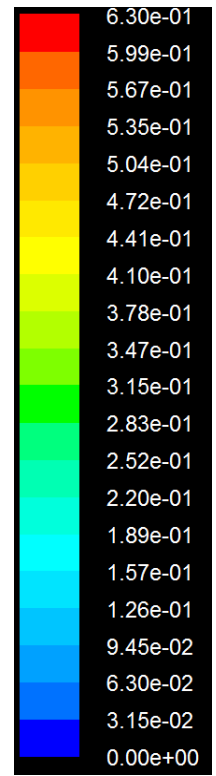

$u=$

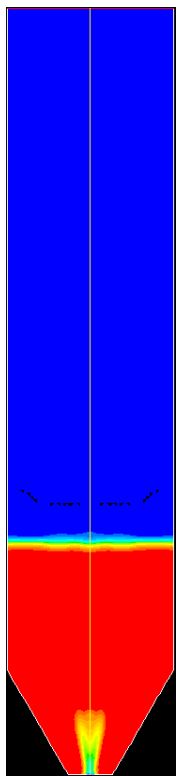

$1 \mathrm{~m} / \mathrm{s}$

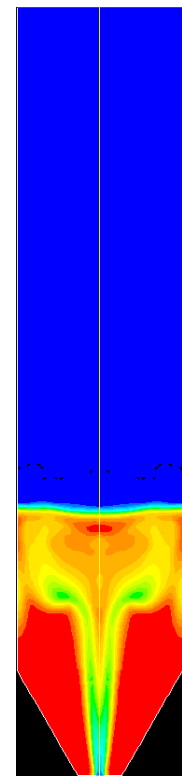

$2.5 \mathrm{~m} / \mathrm{s}$

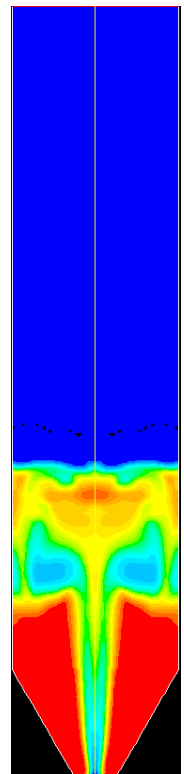

$4 \mathrm{~m} / \mathrm{s}$

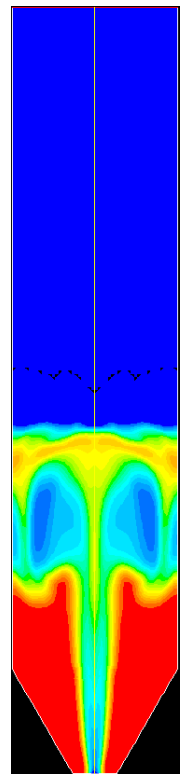

$5.5 \mathrm{~m} / \mathrm{s}$

Figure 8: Contours of the solid volume fraction for different superficial gas velocities ( $\lambda=6 \mathrm{~mm}, H_{b}=21 \mathrm{~cm}$ and $d_{p}=2.28 \mathrm{~mm}$ ).

In the case of an internal jet, a submerged cavity or jet was formed at the air inlet orifice, while the rest of the bed remained as a fixed bed. In the spouting flow regime, the bed had the appearance of a conventional spouted bed. Particles were transported individually by the gas flowing upward in the center of the column and moving downward in the outer regions of the bed. In the incoherent spouting regime, the solids in the spout seemed to be entrained in a periodic manner, with a higher frequency in the upper part of the spout. Particles were more likely to be transported in the spout as small aggregates than individually. The solids in the upper part of the annulus moved downward intermittently. The fountain above the bed surface showed a pulsating variable height. The jet-influidized-bed regime occurred after the formation of the internal jet, during which an increase in the air flow rate led to fluidization of the upper part of the bed. The transition regime had mixed characteristics of the jet-in-fluidized-bed and incoherent spouting flow regimes [6]. 


\section{Conclusion}

Simulations of the gas-solid two-phase flow in a slot-rectangular spouted bed were performed using a computational fluid dynamics technique. The two-phase Eulerian-Eulerian approach was applied. FLUENT commercial software was used for two-dimensional computer simulation, in order to investigate the effects of physical parameters on the hydrodynamic behavior of the bed. The predicted numerical results of the fountain height showed good agreement with earlier experimental data. The effects of the bed height, slot width, solid particle size and superficial gas velocity on the fountain height was studied thoroughly. The fountain height had increasing trend with increases in slot width at constant values of superficial air velocity. The fountain height had a decreasing trend with increases in static bed height for low values of a constant superficial air velocity, and vice versa. The fountain height decreased with increasing particle diameter at constant values of superficial air velocity.

\section{References}

[1] He, Y.L., Qin, S.Z., Lim, C.J. Grace, J.R., "Particle Velocity Profiles and Solid Flow Patterns in Spouted Beds”, Can. J. Chem. Eng. (1994), 72(4), 561-568.

[2] Lim, C.J., Grace, J.R., "Spouted bed hydrodynamics in a $0.91 \mathrm{~m}$ diameter vessel”, Can. J. Chem. Eng. (1987), 65(3), 366-372.

[3] Kalwar, M.I., Raghavan, G.S.V., Mujumdar, A.S., "Spouting of TwoDimensional Beds With Draft Plates”, Can. J. Chem. Eng. (1992), 70(5), 887-894.

[4] Kalwar, M.I., Raghavan, G.S.V., Mujumdar, A.S., “Circulation of Particles in Two-Dimensional Spouted Beds With Draft Plates”, Powder Technol. (1993), 77(3), 233-242.

[5] Passos, M.L., Mujumdar, A.S., Raghavan, G.S.V., "Prediction of the Maximum Spoutable Bed Height in Two-Dimensional Spouted Beds”, Powder Technol. (1993), 74(2), 97-105.

[6] Dogan, O.M., Freitas, L.A.P., Lim, C.J., Grace, J.R., Luo, B., "Hydrodynamics and Stability of Slot-Rectangular Spouted Beds. Part I: Thin Bed”, Chem. Eng. Comm. (2000), 181(1), 225-242.

[7] Freitas, L.A.P., Dogan, O.M., Lim, C.J., Grace, J.R., Luo, B., "Hydrodynamics and Stability of Slot-Rectangular Spouted Beds. Part II: Increasing Bed Thickness”, Chem. Eng. Comm. (2000), 181(1), 243-258.

[8] Dogan, O.M., Uysal, B.Z., Grace, J.R., "Hydrodynamic Studies in a Half Slot-Rectangular Spouted Bed Column”, Chem. Eng. Comm. (2004), 191(4), 566-579.

[9] Chen, Z., Lim, C.J., Grace, J.R., "Hydrodynamics of Slot-Rectangular Spouted Beds-Effect of Slot Configuration on the Local Flow Structure”, Can. J. Chem. Eng. (2008), 86(3), 598-604. 
[10] Freitas, L.A.P., Dogan, O.M., Lim, C.J., Grace, J.R., Bai, D., "Identification of Flow Regimes In Slot-Rectangular Spouted Beds Using Pressure Fluctuations”, Can. J. Chem. Eng. (2004), 82(1), 60-73.

[11] Freitas, L.A.P., Mitsutani, K., Lim, C.J., Grace, J.R., Wei, W., "Voidage Profiles in a Slot-Rectangular Spouted Bed”, Can. J. Chem. Eng. (2004), 82(1), 74-82.

[12] Luo, B., Lim, C.J., Freitas, L.A.P., Grace, J.R., "Flow Characteristics in Slot-Rectangular Spouted Beds with Draft Plates”, Can. J. Chem. Eng. (2004), 82(1), 83-88.

[13] Chen, Z., Lim, C.J., Grace, J.R., "Stability of Slot-Rectangular Spouted Beds: Effect of Slot Configuration”, Can. J. Chem. Eng. (2011), 89(6), 1401-1407.

[14] Deen, N.G., Annaland, M.V., Van der Hoef, M.A., Kuipers, J.A.M., "Review of Discrete Particle Modeling of Fluidized Bed", Chem. Eng. Sci. (2007), 62(1-2), 28-44.

[15] Zhonghua, W., Mujumdar, A.S., "Simulation of the Hydrodynamics and Drying in a Spouted Bed Dryer”, Drying Technol. (2007), 25(1), 59-74.

[16] Zhonghua, W., Mujumdar, A.S., "CFD modeling of the gas-particle flow behavior in spouted beds”, Powder Technology (2008), 183(2), 260-272.

[17] Du, W., Bao, X.J., Xu, J., Wei, W.S., "Computational fluid dynamics (CFD) modeling of spouted bed: Assessment of drag coefficient correlations”, Chem. Eng. Sci. (2006), 61(5), 1401-1420.

[18] Zhao, X.L., Li, S.Q., Liu, G.Q., Song, Q., Yao, Q., “Flow patterns of solids in a two-dimensional spouted bed with draft plates: PIV measurement and DEM simulations”, Powder Technol. (2008), 183(1), 79-87.

[19] Zhao, X.L., Li, S.Q., Liu, G.Q., Yao, Q., Marshall, J.S., “DEM simulation of the particle dynamics in two-dimensional spouted beds”, Powder Technol. (2008), 184(2), 205-213.

[20] Esmaili, E., “Adjustment of Drag Coefficient Correlations in Three Dimensional CFD Simulation of Gas-Solid Bubbling Fluidized Bed”, M.Sc. Thesis (2009), Dept. of Chem. and Petroleum Eng., University of Calgary. 